\title{
Characterization and pathogenicity of soil borne pathogens inducing root rot symptoms in common bean in Rwanda
}

\author{
Mukamuhirwa Floride. ${ }^{1}$, Butare Lewis. ${ }^{1}$, Ruhakana Albert. ${ }^{1}$, Uzayisenga Bellancile. ${ }^{1}$, \\ Mukantwali Christine. ${ }^{1}$, Musoni Augustin. ${ }^{1}$, Mukankusi C. ${ }^{2}$ \\ ${ }^{1}$ Rwanda Agriculture and Animal Resources Development Board, P.O. Box 5016, Kigali Rwanda \\ ${ }^{2}$ International Centre for Tropical Agriculture (CIAT)-Pan Africa Bean Research Alliance (PABRA), P. O. \\ Box 6247, Kampala, Uganda
}

\begin{abstract}
:
Though Rwanda is among the highest common bean producers and consumers, this crop is still challenged by different factors including root rot diseases resulting in low yield potential. Root rot diseases can cause up to $100 \%$ of yield loss. Despite different soil borne pathogens causing bean root rot diseases; there is no recent literature that clearly documents the most aggressive ones affecting common beans in Rwanda. This study aimed at identifying the root rot pathogens affecting bean crop in Rwanda. A survey was conducted to collect bean samples showing root rot symptoms in 12 agro -ecological zones where bean crop is mostly grown. The selection of samples was based on the level of contamination and the purity of cultures. A total number of 132 samples were therefore selected and considered for further analysis. The pathogen isolation was done in Rubona at Rwanda Agriculture and Animal Resources Development Board (RAB) Biotechnology and Pathology laboratory. Structural characterization of soil borne pathogens was also carried out along with pathogenicity evaluation. The growth rate of identified pathogens were significantly different $(\mathrm{P}<0.001)$. Significant differences were observed among isolates inoculated on both plantlets and seeds $(\mathrm{P}<001)$. Four root rot diseases were identified and characterized including Rhizoctonia spp, Fusarium spp,Sclerotium spp and several Pythium spp. The four pathogens were mostly observed in Northern and Southern parts of Rwanda than East and Western parts of Rwanda. Morphological characterization showed that Fusarium spp, Rhizoctonia spp and Sclerotium spp were predominant than Pythium species. Fusarium spp was identified as the most predominant fungal pathogen while Sclerotium spp was the most virulent pathogen. The present study suggests bean root rot disease control program to reduce losses associated with these diseases especially the use of resistant varieties since the fungi are widespread and survive in soils for a long period of time.
\end{abstract}

Key words: Rhizoctonia spp, Fusarium spp, Sclerotium spp, Pythium spp

\section{Introduction}

Common bean (Phaseolus vulgaris L.) is the most important grain legume for direct human consumption in the world. Bean consumption is estimated to more than 300 million people's daily diet [5]. Rwanda is among the highest bean producers and consumers in Sub-Saharan Africa with consumption estimated at $200 \mathrm{~g}$ per capita per day [17]. In some cases, beans are called the meat of the poor due to its high concentration in proteins and its affordability [11],[3] The production in Rwanda is mainly secured by small farmers with average land holdings of less than one hectare and using limited technologies such as limited access to irrigation schemes, limited use of fertilizers and pesticides. Biotic and abiotic factors are the main constraints limiting common bean productivity in Sub-Saharan African countries [2] [17] [17]. Root rot disease caused by soil borne pathogens is among the biotic constraints affecting bean productivity. A number of these soil borne pathogens has been identified in Latin America and Africa such as Fusarium solani f.sp. Phaseoli, Pythium spp, Rhizoctonia solani, Macrophomina phaseoli and Sclerotium rolfsii [6] 
Root rot diseases are most damaging when farming conditions are poor; including water logging, poor drainage capacity, intensification of the cultivation of beans, decline in soil fertility with absence of rotation and use of susceptible varieties [14]. The bean production system in Rwanda is characterized by series of different specific conditions where common bean varieties are grown under high demographic pressure, poor soils with very limited use of crop rotation[10] [18].

\section{Materials and methods}

2.1. The study location and sampling methodology

The study was conducted in all 12agro-ecological zones of Rwanda where bean is grown. Samples were collected in common bean fields showing above ground root rot symptoms such as low plant vigor, yellowing and stunting. A total of 450 bean fields were visited. Three sectors were selected in each district and five bean fields in each sector were surveyed, considering the distance of $2 \mathrm{~km}$ from one field to another. In each bean field, five quadrants each measuring $1 \mathrm{~m}^{2}$ were randomly designed and bean plants showing root rot symptoms were collected from them. A total of 2118 bean samples were collected and samples that did not show any growth of mycelia were discarded along with samples that showed visual contamination with nontargeted pathogens. The disease plant was completely uprooted and put in labeled paper bags. In each sampled field, administrative and geographical information (district, sector, cell, village, altitude, and longitude, latitude, cropping system and soil type) were recorded. One hundred and thirty two samples were then brought to the laboratory for pathogen isolation.

\subsection{Preparation of the media}

Different types of culture media such as Potato Dextrose Agar (PDA), Corn Meal Agar (CMA) and Tap Water Agar (TWA) were used for fungal isolation and growth.

\subsubsection{Preparation of tap water agar}

Sixteen grams of agar extra-pure were added in $1000 \mathrm{ml}$ of tap water, shaken until the solution reaches the homogeneity stage and autoclaved at $121^{\circ} \mathrm{C}$ for 20 minutes. The autoclaved media was allowed to cool down to $40^{\circ} \mathrm{Cunder}$ laminar flow then dispensed in $9 \mathrm{~mm}$ wide petri dishes in the rate of $20 \mathrm{ml}$ per petri dish. The collected samples were prepared into piece of roots, isolated on tap water agar and incubated at $23^{\circ} \mathrm{C}$ for 24 hours. Pathogen
Symptoms and severity of root rot diseases depend on the environment conditions including rainfall, moisture, temperature, soil type, drainage, cropping system, and the interaction between responsible pathogens. The objective of this study was to identify and characterize root rot diseases caused by soil borne pathogens of common bean (Phaseolus vulgaris) in Rwanda.

isolation was performed using a laboratory protocol as described in the International Center for Tropical Agriculture (CIAT) training manual (Mukamuhirwa et al., 2017). All emerging fungi from the roots cultured on tap water agar were sub cultured on PDA and CMA.

\subsubsection{Preparation of Potato dextrose Agar and Corn Meal Agar}

Sixteen grams of PDA or CMA were added to 1000 $\mathrm{ml}$ of distilled sterilized water. The mixtures were shaken until the solutions reach the homogeneity stage and autoclaved at $121^{\circ} \mathrm{C}$ for 20 minutes. The autoclaved media was allowed to cool down to $40^{\circ} \mathrm{C}$ under laminar flow then dispensed in $9 \mathrm{~mm}$ wide petri dishes in the rate of $20 \mathrm{ml}$ per petri dish. A bock agar from tap water media was transferred to the petri dishes with PDA to identify Rhizoctonia spp, Sclerotium spp and Fusarium spp while CMA was used to identify Pithium spp from other root rot pathogens. The petri dishes were incubated at $23^{\circ} \mathrm{C}$ [14].

\subsection{Pathogens isolation}

The pathogen isolation was carried out in the Rwanda Agriculture and Animal Resources Development Board (RAB), Biotechnology and Pathology Laboratories. A single hyphal isolation was conducted under microscope on mycelium tips. All isolates were transferred on fresh medium of PDA and CMA with sterilized filter paper (Waltman paper $\mathrm{n}^{\mathrm{o}}$ ) for root rot pathogens long term conservation. Filter paper was cut into small pieces of about $4 \mathrm{~cm}$ squared. The pieces of paper were then folded into aluminium paper and sterilized at $121^{\circ} \mathrm{C}$ for 20 minutes. The pieces of paper were cooled down to room temperature. Using sterilized forceps under laminar flow, about 20 sterilized pieces of filter papers were placed on PDA and/ or CMA containing colonized agar block to allow the pathogens to grow and colonize the filter papers. Colonized filter papers were then transferred to new sterilized empty petri dishes. These petri dishes were 
placed into sterilized desiccators for two to three days to allow the filter papers to dry. The dried colonized filter papers were removed and wrapped in sterile Aluminum foil, clearly labeled and stored under $-20^{\circ} \mathrm{C}$ in freezer for further use.

\subsection{Pathogens isolation}

The pathogen isolation was carried out in the Rwanda Agriculture and Animal Resources Development Board (RAB), Biotechnology and Pathology Laboratories. A single hyphal isolation was conducted under microscope on mycelium tips. All isolates were transferred on fresh medium of PDA and CMA with sterilized filter paper (Waltman paper $n^{0}$ ) for root rot pathogens long term conservation. Filter paper was cut into small pieces

\subsection{Structural characterization of soil borne pathogens}

Cultures were initiated for the growth rate measurements and phenotypic description of different pathogens. The description of the different root rot pathogens was done using their colony texture, mycelia color, presence or absence of sclerotia, color of sclerotia, colony margin and number of sclerotia, clear zonation and mycelia floral patterns. Microscopic identification of the pathogens was also done basing on the presence of micro and macro conidia (Fusarium spp.), and the hyphal branching at the perpendicular angles or Tshaped branching (Rhizoctonia spp.). Colony diameters for all cultures initiated for growth rate variations were measured after 24 hours of incubation. This was done by drawing two perpendicular lines at the base of the petri-dish and colony margins measured using a ruler along the lines. Measurements were carried on every day until when the plates were completely covered.

\subsection{Pathogenicity evaluation}

The pathogenicity test of isolated pathogens was performed using 132 isolates. The sorghum grain media was used for fungal inoculum production. Approximately $400 \mathrm{ml}$ of distilled water were added to every $300 \mathrm{~g}$ of sorghum grains, loaded into a sterilized glass bottle, the autoclaved twice at $121^{\circ} \mathrm{C}$ for $20 \mathrm{~min}$ and allowed to cool for 12 hours. The sterilized sorghum was then inoculated with the liquid suspension of $100 \mu \mathrm{l}$ scraped pathogen mycelia dissolved in sterile distilled water. The media substrate bottles were hand shaken daily to homogenize the fungal distribution and growth. The inoculum was incubated in the bottles over the sterilized sorghum grains in a sterile environment at of about $4 \mathrm{~cm}$ squared. The pieces of paper were then folded into aluminium paper and sterilized at $121^{\circ} \mathrm{C}$ for 20 minutes. The pieces of paper were cooled down to room temperature. Using sterilized forceps under laminar flow, about 20 sterilized pieces of filter papers were placed on PDA and/ or CMA containing colonized agar block to allow the pathogens to grow and colonize the filter papers. Colonized filter papers were then transferred to new sterilized empty petri dishes. These petri dishes were placed into sterilized desiccators for two to three days to allow the filter papers to dry. The dried colonized filter papers were removed and wrapped in sterile Aluminum foil, clearly labeled and stored under $-20^{\circ} \mathrm{C}$ in freezer for further use

room temperature for 14 days to allow uniform growth[4] [7]. Pathogenicity evaluation was done through 3 different experiments while the seed rot experiment was conducted in petri dishes following the MSU protocol where the colonized agar block was placed in the middle of fresh media and allowed to grow and infect the 10 sterilized healthy seed in the petri dish. The incubation was done for 7 days and the disease severity was assessed using 1-5 scale where 1: Germinated, 2: Delayed germination; 3: Germination and some Lesions; 4: Germination with coalesced lesions; 5: Seed colonized. The second experiment was conducted on laboratory bench where plastic boxes where used to plant sterilized healthy seed on sterilized paper towel. Bean seed were allowed to grow for 2 weeks, taking care of removal of any plant showing the symptoms of the disease. This was followed by inoculation of roots of plantlets with colonized sorghum grain, incubate for 7 days as explained above and evaluate the severity of the disease using 1-5CIAT scale. The third experiment was set in pots of 5liters in screen house following CIAT Training manual.

\subsection{Statistical analysis}

The slope between the first and last day of measurement was subjected to Analysis of Variance (ANOVA). The Disease Index (DI) scored on seed rot experiment was calculated as: $\mathrm{DI}=$ $\Sigma$ (severity $\mathrm{X} n$ ) / $\mathrm{N}$ [8] and the means were alsosubjected to ANOVA. Disease severity for plantlet inoculation in laboratory bench and screen house data in pots were recorded following CIAT scale and subjected to ANOVA.All the analysis were performed using Gen-stat Statistical Package.

\section{Results}




\subsection{Morphological characteristics}

and distribution of root rot diseases in Rwanda

The colony type of identified isolates was $69 \%$ cotton and $31 \%$ flat. The regular and or round colony margin was observed at $43 \%$ while the irregular colony margin was observed at $57 \%$. The mycelia of the identified pathogens were raised at $70 \%$ and thin at $30 \%$. From the identified pathogens the mycelia color was $36 \%$ purple, $14 \%$ red, $25 \%$ cream and $25 \%$ white. The growth rate of identified pathogens were significantly different $(\mathrm{P}<0.001)$ with the range of slope varying between 0.1 to 5.3 $\mathrm{mm}$ (Table 1). How many isolates were classified as fast growing, slow or medium?Among the 132 isolates, 68\%, 9\%, 32\%, and 30\% wereFusarium spp, Pythium spp, Rhizoctonia spp, and Sclerotium spp respectively. Morphological characterization showed that Fusarium, Rhizoctonia and Sclerotium isolates were more predominant than Pythium species (Figure 1).

Figure 1: Bean root rot pathogens identified in Rwanda.

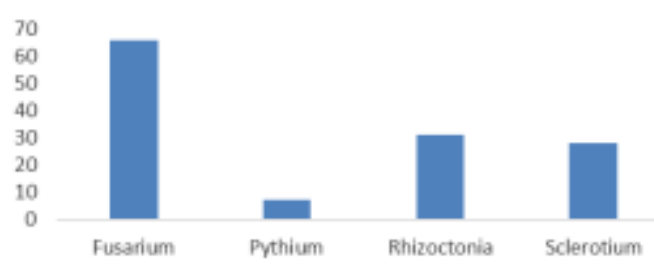

\begin{tabular}{|l|l|l|l|l|l|}
\hline Change & DF & SS & MS & v.r. & F pr. \\
\hline Replication & 2 & 0.322 & 0.2 & 0.2 & 0.9 \\
\hline Sample ID & 131 & 505.3 & 3.8 & 2.7 & $1.0 \mathrm{E}-17$ \\
\hline Residual & 788 & 1108.2 & 1.4 & & \\
\hline \%CV & 23.7 & & & & \\
\hline SEM & 0.3 & & & & \\
\hline
\end{tabular}

Table 1. Analysis of variance for slope of bean root rot diseases identified in different sites

\section{2. Pathogenic characterization}

The most virulent isolates were selected for screening varieties for resistance to root rot pathogens. Selection of virulent isolate was done in 3 experiments which showed the same results. The study identified a high level of diversity in bean root rot diseases between (Figure 3:F1) and within species (Figure 3:F2 and F3). The four diseased were observed especially in Figure 1. The virulence study showed that isolates from southern and western locations were more virulent than the rest.
Three most virulent and aggressive isolates were selected including Sclerotium 63 isolate collected from the District of Kamonyi in Runda sector, 84 Rhizoctonia 84 collected from Ruhango District, Byimana sector and Fusarium 196 collected from Southern Province of Rwanda (Table 2 and Figure 2). The Greenhouse experiment confirmed the results of seed rot assay and plantlet rot experiments (Table 3). The most aggressive isolates selected based on all experiments.

Figure 2: Map of the study area

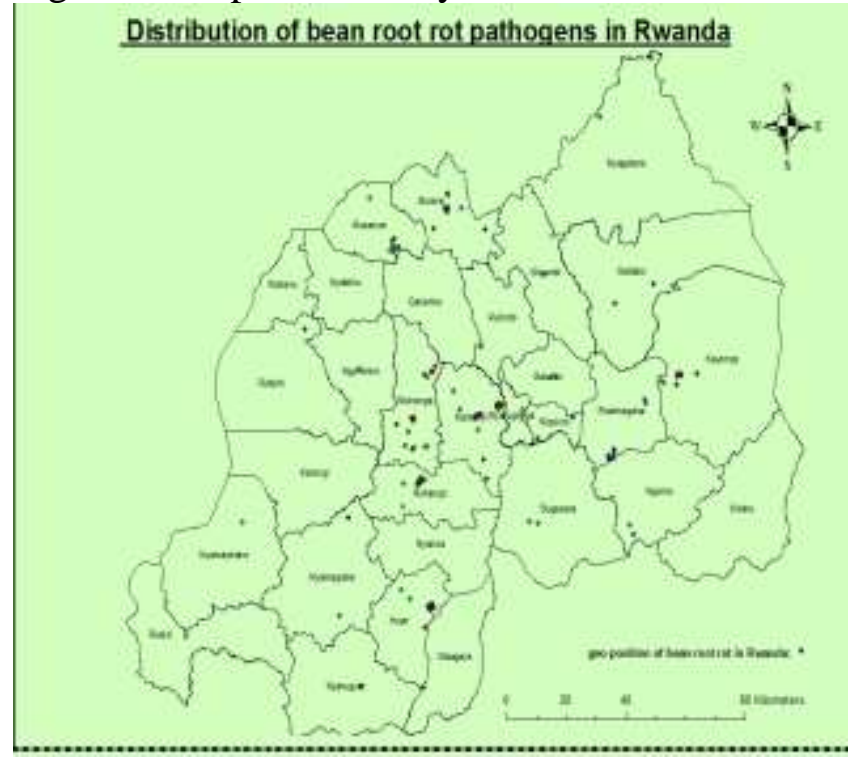

Figure 3: Pathogen isolation on Tap Water Agar (A), Subculture on Potato Dextrose Agar (B) Single hyphal tip under microscopic observation (C), pure culture (D) growth measurement (E1 at the first day \&E2 at the last day) and colony features assessment and diversity (F1: the four pathogens identified, F2 diversity in Rhizoctonia spp, F3 diversity in Sclerotium spp).

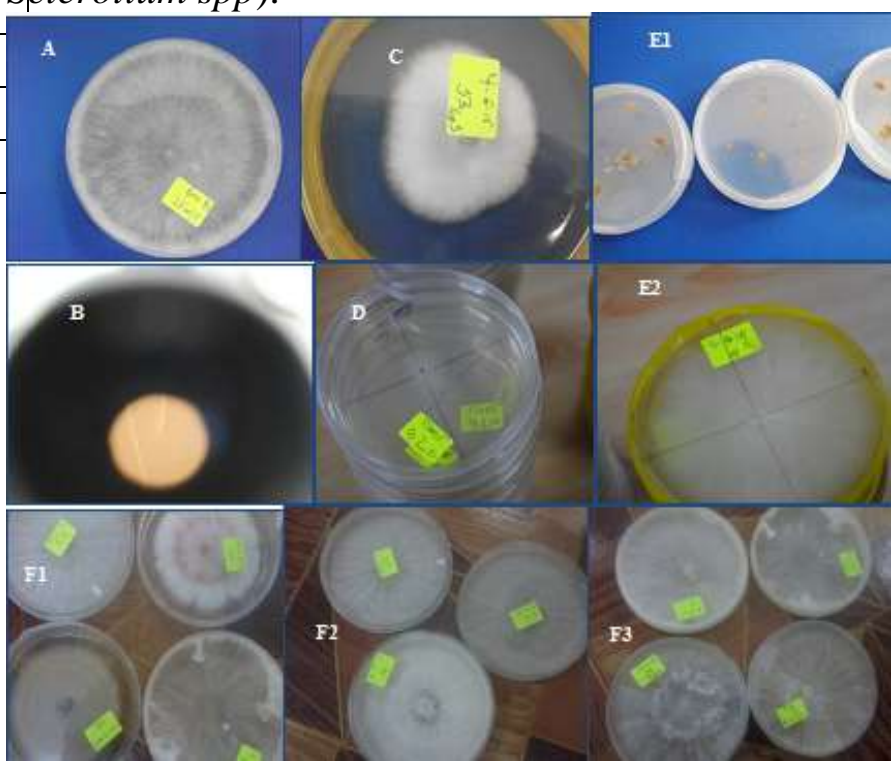




\begin{tabular}{|c|c|c|c|c|c|c|c|c|c|}
\hline 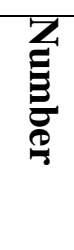 & 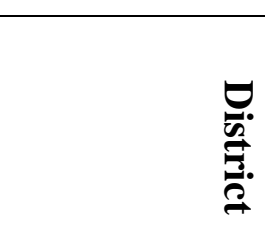 & $\Xi$ & 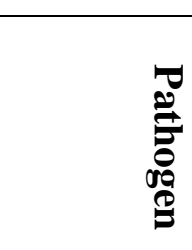 & 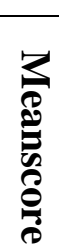 & $\underset{\Xi}{Z}$ & 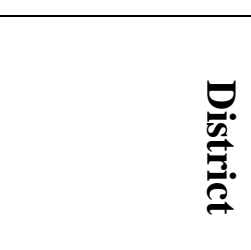 & $\Xi$ & 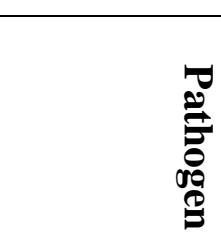 & 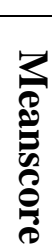 \\
\hline 1 & Muhanga & 33 & Fusarium & 2 & 67 & Muhanga & $67-1$ & Fusarium & 2 \\
\hline 2 & Muhanga & 124 & Fusarium & 4 & 68 & Kayonza & $45-2$ & Fusarium & 2 \\
\hline 3 & Kamonyi & $62-1$ & Fusarium & 1 & 69 & Huye & 158 & Fusarium & 2 \\
\hline 4 & Kamonyi & 144 & Fusarium & 4 & 70 & Ruhango & 10 & Fusarium & 3 \\
\hline 5 & Kamonyi & 184 & Fusarium & 1 & 71 & Ruhango & 213 & Fusarium & 2 \\
\hline 6 & Kamonyi & $* 1-2$ & Fusarium & 2 & 72 & Ruhango & 75 & Fusarium & 2 \\
\hline 7 & Kamonyi & $* 1-1$ & Fusarium & 1 & 73 & Bugesera & 210 & Fusarium & 2 \\
\hline 8 & Gatsibo & 220 & Fusarium & 2 & 74 & Muhanga & $41-1$ & Fusarium & 1 \\
\hline 9 & Burera & 141 & Fusarium & 1 & 75 & Muhanga & 125 & Fusarium & 2 \\
\hline 10 & Kamonyi & 3 & Fusarium & 1 & 76 & Muhanga & 171 & Fusarium & 3 \\
\hline 11 & Kamonyi & 129 & Fusarium & 2 & 77 & Muhanga & 221 & Fusarium & 2 \\
\hline 12 & Kamonyi & 72 & Fusarium & 2 & 78 & Ruhango & 11 & Fusarium & 2 \\
\hline 13 & Rwamagana & 47 & Fusarium & 4 & 79 & Kamonyi & 58 & Fusarium & 2 \\
\hline 14 & Rwamagana & $47-1$ & Fusarium & 3 & 80 & Gatsibo & 185 & Fusarium & 2 \\
\hline 15 & Kayonza & 39 & Fusarium & 1 & 81 & Kicukiro & $182-2$ & Fusarium & 2 \\
\hline 16 & Kamonyi & 1 & Fusarium & 2 & 82 & Gatsibo & 185 & Fusarium & 3 \\
\hline 17 & Kamonyi & 201 & Fusarium & 1 & 83 & Gatsibo & 218 & Fusarium & 3 \\
\hline 18 & Kamonyi & 121 & Fusarium & 2 & 84 & Gicumbi & 203 & Fusarium & 3 \\
\hline 19 & Kicukiro & 217 & Fusarium & 2 & 85 & Rwamagana & 188 & Fusarium & 3 \\
\hline 20 & Kicukiro & 182 & Fusarium & 3 & 86 & Rwamagana & inconue & Fusarium & 2 \\
\hline 21 & BUGESERA & 126 & Fusarium & 3 & 87 & Kamonyi & 63 & Sclerotium & 5 \\
\hline 22 & Nyagatare & 187 & Fusarium & 3 & 88 & Muhanga & 51 & Sclerotium & 5 \\
\hline 23 & Nyamagabe & 200 & Fusarium & 3 & 89 & Kamonyi & 32 & Sclerotium & 5 \\
\hline 24 & Kayonza & 29 & Fusarium & 4 & 90 & Huye & 94 & Sclerotium & 5 \\
\hline 25 & Musanze & 111-1 & Fusarium & 3 & 91 & Kayonza & 20 & Sclerotium & 4 \\
\hline 26 & Musanze & 14 & Fusarium & 3 & 92 & Kayonza & 31 & Sclerotium & 4 \\
\hline 27 & Muhanga & 6 & Fusarium & 4 & 93 & Huye & 90 & Sclerotium & 5 \\
\hline 28 & Muhanga & 6 & Fusarium & 4 & 94 & Huye & 95 & Sclerotium & 5 \\
\hline 29 & Burera & 146 & Fusarium & 3 & 95 & Huye & 89 & Sclerotium & 5 \\
\hline 30 & Huye & $202-2$ & Fusarium & 1 & 96 & Kamonyi & 4 & Sclerotium & 5 \\
\hline 31 & Kamonyi & 24 & Fusarium & 2 & 97 & Muhanga & 71 & Sclerotium & 5 \\
\hline 32 & Muhanga & $67-1$ & Fusarium & 2 & 98 & Kayonza & 68 & Sclerotium & 5 \\
\hline 33 & Muhanga & $67-1$ & Fusarium & 2 & 99 & Kayonza & 76 & Sclerotium & 5 \\
\hline 34 & Burera & $148-1$ & Fusarium & 2 & 100 & Kamonyi & 19 & Sclerotium & 4 \\
\hline 35 & Rwamagana & 191 & Fusarium & 2 & 101 & Ruhango & 14 & Sclerotium & 4 \\
\hline 36 & Ruhango & 12 & Fusarium & 3 & 102 & Huye & 91 & Sclerotium & 4 \\
\hline 37 & Musanze & $115-2$ & Fusarium & 2 & 103 & Muhanga & 50 & Sclerotium & 5 \\
\hline 38 & Ruhango & 166 & Fusarium & 1 & 104 & Kamonyi & 60 & Sclerotium & 4 \\
\hline 39 & Ngororero & 139 & Fusarium & 1 & 105 & Kamonyi & 63 & Sclerotium & 5 \\
\hline 40 & Kamonyi & 174 & Fusarium & 3 & 106 & Nyamasheke & 132 & Sclerotium & 4 \\
\hline
\end{tabular}




\begin{tabular}{|c|c|c|c|c|c|c|c|c|c|}
\hline 41 & Burera & 145 & Fusarium & 3 & 107 & Rutsiro & 205 & Sclerotium & 3 \\
\hline 42 & Burera & 131 & Fusarium & 3 & 108 & Huye & 85 & Sclerotium & 4 \\
\hline 43 & Burera & 219 & Fusarium & 4 & 109 & Kamonyi & 69 & Rhizoctonia & 3 \\
\hline 44 & Kayonza & 181 & Fusarium & 3 & 110 & Kayonza & 31 & Rhizoctonia & 1 \\
\hline 45 & Muhanga & 127 & Fusarium & 1 & 111 & Kayonza & 31 & Rhizoctonia & 4 \\
\hline 46 & Kamonyi & 117 & Fusarium & 2 & 112 & Ruhango & 84 & Rhizoctonia & 3 \\
\hline 47 & Nyamagabe & 173 & Fusarium & 1 & 113 & Ruhango & 84 & Rhizoctonia & 4 \\
\hline 48 & Gasabo & 156 & Fusarium & 3 & 114 & Nyarugenge & 124 & Rhizoctonia & 1 \\
\hline 49 & BURERA & 140 & Fusarium & 3 & 115 & Kayonza & 21 & Rhizoctonia & 3 \\
\hline 50 & Burera & 151 & Fusarium & 3 & 116 & Ruhango & 82 & Rhizoctonia & 4 \\
\hline 51 & Nyaruguru & 196 & Fusarium & 4 & 117 & Rwamagana & 183 & Rhizoctonia & 3 \\
\hline 52 & Burera & 147 & Fusarium & 2 & 118 & Ruhango & 17 & Rhizoctonia & 4 \\
\hline 53 & Huye & 87 & Fusarium & 2 & 119 & Ruhango & 10 & Rhizoctonia & 4 \\
\hline 54 & Gatsibo & 189 & Fusarium & 3 & 120 & Kamonyi & $62-2$ & Rhizoctonia & 3 \\
\hline 55 & Huye & $202-1$ & Fusarium & 2 & 121 & Musanze & 97 & Rhizoctonia & 3 \\
\hline 56 & Ruhango & 55 & Fusarium & 2 & 122 & Musanze & 106 & Rhizoctonia & 4 \\
\hline 57 & Musanze & $113-1$ & Fusarium & 2 & 123 & Ruhango & 16 & Rhizoctonia & 5 \\
\hline 58 & Burera & $142-1$ & Fusarium & 3 & 124 & Musanze & 102 & Rhizoctonia & 4 \\
\hline 59 & Gisagara & 175 & Fusarium & 2 & 125 & Kamonyi & 48 & Rhizoctonia & 4 \\
\hline 60 & Huye & 216 & Fusarium & 2 & 126 & Ngoma & RG2-1 & Pythium & 3 \\
\hline 61 & Muhanga & $74-2$ & Fusarium & 2 & 127 & Burera & RG3-4 & Pythium & 2 \\
\hline 62 & Huye & 88 & Fusarium & 2 & 128 & Kicukiro & RG4-3 & Pythium & 3 \\
\hline 63 & Rwamagana & $191-1$ & Fusarium & 1 & 129 & Kicukiro & RG4-3 & Pythium & 2 \\
\hline 64 & Muhanga & 38 & Fusarium & 1 & 130 & Ngoma & RG5-6 & Pythium & 3 \\
\hline 65 & Ruhango & 198 & Fusarium & 2 & 131 & Musanze & RG6-8 & Pythium & 2 \\
\hline 66 & Rulinndo & 199 & Fusarium & 2 & 132 & Rwamagana & RG7-4 & Pythium & 3 \\
\hline
\end{tabular}

Table 2:.Identification of root rots pathogens inducing root rot symptoms

\begin{tabular}{|c|c|c|c|c|c|}
\hline Change & DF & SS & MS & v.r. & F pr. \\
\hline Rep & 2 & 0.27966 & 0.13983 & 1.214153 & \\
\hline Isolate & 131 & 211.0758 & 1.611266 & 13.99072 & $<0.001$ \\
\hline Residual & 262 & 30.17368 & 0.115167 & & \\
\hline Total & 395 & 241.5292 & & & \\
\hline CV & & & 9 & & \\
\hline LSD & & & 0.5012 & & \\
\hline
\end{tabular}

Analysis of variance of bench method

\begin{tabular}{|c|c|c|c|c|c|}
\hline Change & DF & SS & MS & v.r. & F pr. \\
\hline Rep & 2 & 284 & 142 & 1444.051 & \\
\hline Isolate & 131 & 360.5104 & 2.751988 & 27.986 & $<.001$ \\
\hline Residual & 262 & 25.76363 & 0.098334 & & \\
\hline Total & 395 & 670.274 & 1.696896 & & \\
\hline \% CV & & & 11.58 & & \\
\hline LSD & & & 0.4451 & & \\
\hline
\end{tabular}

Analysis of Variance of screen house experiment

\begin{tabular}{|c|c|c|c|c|c|}
\hline Change & DF & SS & MS & v.r. & F pr. \\
\hline Rep & 2 & 0.2797 & 0.1399 & 0.922795 & 0.3987 \\
\hline Isolate & 131 & 201.5433 & 1.5385 & 10.15173 & $<.001$ \\
\hline
\end{tabular}




\begin{tabular}{|c|c|c|c|l|l|}
\hline Residual & 262 & 39.7062 & 0.1516 & & \\
\hline Total & 395 & 234.248 & & & \\
\hline \% CV & 12.84 & & & & \\
\hline LSD & 0.5628 & & & & \\
\hline
\end{tabular}

Table 3: Analysis of variance of seed rot assay

\begin{tabular}{|c|c|c|c|c|c|c|c|c|}
\hline$\Xi \frac{\mathscr{E}}{\tilde{E}}$ & 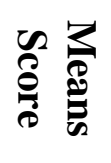 & $\stackrel{\Omega}{0}$ & $\exists \frac{\mathscr{E}}{\stackrel{\mathscr{E}}{0}}$ & 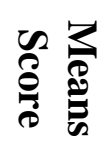 & $\stackrel{\Omega}{0}$ & 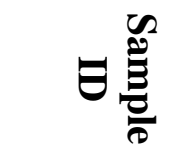 & 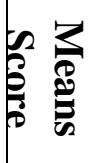 & $\frac{\Omega}{0}$ \\
\hline 11 & 5 & $\mathrm{~A}$ & 71 & 5 & $a b$ & 188 & 4 & defghijklm \\
\hline 4 & 5 & $\mathrm{~A}$ & 38 & 5 & $a b c$ & 189 & 4 & defghijklm \\
\hline 13 & 5 & $\mathrm{~A}$ & 81 & 4 & bcd & 203 & 4 & defghijklm \\
\hline 16 & 5 & $\mathrm{~A}$ & 133 & 4 & cde & 218 & 4 & defghijklmn \\
\hline 19 & 5 & $\mathrm{~A}$ & 183 & 4 & cdef & $111-1$ & 4 & defghijklmn \\
\hline 20 & 5 & A & $131-1$ & 4 & cdef & 120 & 4 & $\begin{array}{c}\text { defghijklmn } \\
\text { o }\end{array}$ \\
\hline 48 & 5 & $\mathrm{~A}$ & 141 & 4 & cdefg & 167 & 4 & $\begin{array}{c}\text { defghijklmn } \\
\text { o }\end{array}$ \\
\hline 50 & 5 & $\mathrm{~A}$ & 216 & 4 & cdefg & 127 & 4 & $\begin{array}{c}\text { defghijklmn } \\
\text { op }\end{array}$ \\
\hline 51 & 5 & A & 23 & 4 & cdefgh & 90 & 4 & $\begin{array}{c}\text { efghijklmno } \\
\text { pq }\end{array}$ \\
\hline 60 & 5 & $\mathrm{~A}$ & 128 & 4 & cdefgh & 166 & 4 & $\begin{array}{c}\text { efghijklmno } \\
\text { pq }\end{array}$ \\
\hline 68 & 5 & $\mathrm{~A}$ & 221 & 4 & cdefgh & 197 & 4 & $\begin{array}{c}\text { efghijklmno } \\
\text { pq }\end{array}$ \\
\hline 69 & 5 & $\mathrm{~A}$ & 182 & 4 & cdefghi & 220 & 4 & $\begin{array}{c}\text { efghijklmno } \\
\text { pq }\end{array}$ \\
\hline 76 & 5 & A & $67-2$ & 4 & cdefghi & $47-1$ & 4 & $\begin{array}{c}\text { efghijklmno } \\
\text { pq }\end{array}$ \\
\hline 84 & 5 & $\mathrm{~A}$ & 2 & 4 & defghij & $74-2$ & 4 & $\begin{array}{c}\text { efghijklmno } \\
\text { pq }\end{array}$ \\
\hline 88 & 5 & $\mathrm{~A}$ & 75 & 4 & defghij & 87 & 4 & $\begin{array}{c}\text { fghijklmnop } \\
\text { qr }\end{array}$ \\
\hline 89 & 5 & $\mathrm{~A}$ & $142-1$ & 4 & defghij & 146 & 4 & $\begin{array}{c}\text { fghijklmnop } \\
\text { qr }\end{array}$ \\
\hline 94 & 5 & $\mathrm{~A}$ & 125 & 4 & defghijk & 176 & 4 & $\begin{array}{c}\text { fghijklmnop } \\
\text { qr }\end{array}$ \\
\hline 95 & 5 & A & 147 & 4 & defghijkl & 202 & 4 & $\begin{array}{c}\text { fghijklmnop } \\
\text { qr }\end{array}$ \\
\hline 63 & 5 & A & 182 & 4 & defghijkl & $41-1$ & 4 & $\begin{array}{c}\text { fghijklmnop } \\
\text { qr }\end{array}$ \\
\hline 8 & 5 & $\mathrm{~A}$ & 217 & 4 & defghijkl & 35 & 4 & $\begin{array}{c}\text { ghijklmnopq } \\
\text { rs }\end{array}$ \\
\hline
\end{tabular}




\begin{tabular}{|c|c|c|c|c|c|}
\hline$\Xi \frac{\mathscr{\mathscr { E }}}{\bar{E}}$ & 胥 & $\frac{\Omega}{0}$ & 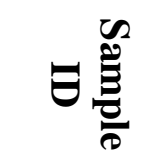 & $\underset{2}{2}$ & $\stackrel{\Omega}{0}$ \\
\hline 140 & 4 & ghijklmnopqrs & 158 & 4 & lmnopqrstuvwxy \\
\hline 144 & 4 & ghijklmnopqrs & $74-1$ & 4 & lmnopqrstuvwxy \\
\hline 151 & 4 & ghijklmnopqrs & 12 & 4 & mnopqrstuvwxy \\
\hline 175 & 4 & ghijklmnopqrs & 163 & 4 & mnopqrstuvwxy \\
\hline 190 & 4 & ghijklmnopqrs & 124 & 4 & nopqrstuvwxyz \\
\hline $115-1$ & 4 & ghijklmnopqrs & 139 & 4 & nopqrstuvwxyz \\
\hline 39 & 4 & hijklmnopqrst & 171 & 4 & nopqrstuvwxyz \\
\hline 132 & 4 & hijklmnopqrst & $23-3$ & 4 & nopqrstuvwxyz \\
\hline 135 & 4 & hijklmnopqrst & 125 & 4 & opqrstuvwxyzA \\
\hline 187 & 4 & hijklmnopqrst & 182 & 4 & opqrstuvwxyzA \\
\hline 206 & 4 & hijklmnopqrst & 185 & 4 & opqrstuvwxyzA \\
\hline 85 & 4 & hijklmnopqrstu & 191 & 4 & opqrstuvwxyzA \\
\hline 191 & 4 & hijklmnopqrstu & 202 & 4 & opqrstuvwxyzA \\
\hline 204 & 4 & ijklmnopqrstuv & & & \\
\hline 118 & 4 & jklmnopqrstuvw & & & \\
\hline 126 & 4 & jklmnopqrstuvw & & & \\
\hline 189 & 4 & jklmnopqrstuvw & & & \\
\hline 64 & 4 & klmnopqrstuvwx & & & \\
\hline 174 & 4 & klmnopqrstuvwx & & & \\
\hline 3 & 4 & lmnopqrstuvwxy & & & \\
\hline
\end{tabular}

Table 4: Means scores of the selected virulent isolates under seed rot assay experiment

\begin{tabular}{|c|c|c|c|c|}
\hline $\begin{array}{c}\text { Sample } \\
\text { ID }\end{array}$ & Disease & Average & Max & Minimum \\
\hline 51 & Sclerotium & 5 & 5 & 3 \\
\hline 32 & Sclerotium & 5 & 5 & 4 \\
\hline 20 & Sclerotium & 4 & 5 & 4 \\
\hline 90 & Sclerotium & 5 & 5 & 3 \\
\hline 95 & Sclerotium & 5 & 5 & 4 \\
\hline 89 & Sclerotium & 5 & 5 & 4 \\
\hline 4 & Sclerotium & 5 & 5 & 4 \\
\hline 71 & Sclerotium & 5 & 5 & 4 \\
\hline 68 & Sclerotium & 5 & 5 & 4 \\
\hline 50 & Sclerotium & 5 & 5 & 4 \\
\hline 63 & Sclerotium & 5 & 5 & 5 \\
\hline 31 & Rhizoctonia & 4 & 5 & 2 \\
\hline 84 & Rhizoctonia & 5 & 5 & 3 \\
\hline 21 & Rhizoctonia & 3 & 5 & 1 \\
\hline 82 & Rhizoctonia & 4 & 5 & 1 \\
\hline 17 & Rhizoctonia & 4 & 5 & 1 \\
\hline 17 & Rhizoctonia & 4 & 5 & 1 \\
\hline 10 & Rhizoctonia & 4 & 5 & 1 \\
\hline 97 & Rhizoctonia & 3 & 5 & 1 \\
\hline
\end{tabular}




\begin{tabular}{|c|c|c|c|c|}
\hline 106 & Rhizoctonia & 4 & 5 & 2 \\
\hline 16 & Rhizoctonia & 5 & 5 & 3 \\
\hline 102 & Rhizoctonia & 4 & 5 & 2 \\
\hline 33 & Fusarium & 2 & 4 & 1 \\
\hline 196 & Fusarium & 4 & 5 & 3 \\
\hline 14 & Fusarium & 4 & 5 & 2 \\
\hline 124 & Fusarium & 4 & 5 & 2 \\
\hline 219 & Fusarium & 4 & 5 & 2 \\
\hline 47 & Fusarium & 4 & 5 & 2 \\
\hline 6 & Fusarium & 4 & 5 & 2 \\
\hline 141 & Fusarium & 4 & 5 & 2 \\
\hline 174 & Fusarium & 3 & 5 & 1 \\
\hline$* 1-2$ & Fusarium & 2 & 5 & 1 \\
\hline 220 & Fusarium & 2 & 4 & 1 \\
\hline 129 & Fusarium & 2 & 4 & 1 \\
\hline 1 & Fusarium & 2 & 4 & 1 \\
\hline 72 & Fusarium & 2 & 4 & 1 \\
\hline
\end{tabular}

Table 5: Means scores of the selected virulent isolates under bench (Plantlet rot) Experiment

\begin{tabular}{ccccc}
\hline $\begin{array}{c}\text { Sample } \\
\text { ID }\end{array}$ & Disease & Average & Max & Minimum \\
51 & Scolerotium & 5 & 5 & 3 \\
32 & Scolerotium & 5 & 5 & 4 \\
20 & Sclerotium & 4 & 5 & 4 \\
90 & Sclerotium & 5 & 5 & 3 \\
95 & Sclerotium & 5 & 5 & 4 \\
89 & Sclerotium & 5 & 5 & 4 \\
71 & Sclerotium & 5 & 5 & 4 \\
68 & Sclerotium & 5 & 5 & 4 \\
50 & Sclerotium & 5 & 5 & 4 \\
63 & Sclerotium & 5 & 5 & 5 \\
84 & Rhizoctonia & 5 & 5 & 3 \\
16 & Rhizoctonia & 5 & 5 & 3 \\
102 & Rhizoctonia & 4 & 5 & 2 \\
33 & Fusarium & 2 & 4 & 1 \\
196 & Fusarium & 4 & 5 & 3 \\
219 & Fusarium & 4 & 5 & 2 \\
174 & Fusarium & 3 & 5 & 1 \\
1 & Fusarium & 2 & 5 & 1 \\
69 & Rhizoctonia & 3 & 4 & 2 \\
94 & Scoleratium & 3 & 5 & 2 \\
19 & Scoleratium & 4 & 5 & 3 \\
218 & Rusarium & 3 & 4 & 3 \\
131 & Fusarium & 3 & 5 & 1 \\
151 & Fusarium & 3 & 5 & 3 \\
121 & Fusarium & 2 & 5 & \\
60 & Scoleratium & 4 & 5 & 5 \\
175 & Fusarium & 3 & 5 & 5 \\
\hline
\end{tabular}




\section{$74 / 2 \quad$ Fusarium $\quad 2 \quad 4 \quad 1$

188 Fusarium 3304 \\ Table 6: Mean scores of the selected virulent isolates under green house experiment, plantlet rot on bench experiment and seed rot experiment in petri dishes}

\section{Discussions}

The isolation protocol used in this study allowed finding out different isolates of bean root rot

diseases. Using morphological characterization, our study showed that Fusarium spp, Rhizoctonia spp and Sclerotium spp were predominant than pythium spp.

CIAT reported that Pythium and Fusarium species are more important particularly under high soil moisture content and low temperatures which favor the root rot disease development [14] reported that the Pythium species identified in Rwanda were pathogenic on common bean. The present study revealed the four pathogens causing bean root rot diseases including Fusarium spp, Pythium spp, Rhizoctonia spp and Sclerotium spp where Fusarium spp is more predominant while Sclerotium spp is more aggressive than the rest. This is consistent with several studies where it was shown that bean root rot diseases are worldwide spread. Fusarium root rot was found to be present wherever dry beans are grown. In his study, [14] revealed the wide distribution of Pythium species in Rwanda. He notified the presence of several Pythium species including $P$. vexansPythium indigoferae, Pythium torulosum, Pythium, ultimum and Pythium rostratifingens, Pythiumcucurbitacearum, Pythium arrhenomanes, Pythium pachycaule and Pythium rostratum.

In the present study, diseased locations were observed especially in northern and southern parts of Rwanda while east and western parts were less affected. Several studies showed that the distribution of bean root rot diseases depend on several factors including species type[14]. The most virulent pathogens were found within Sclerotium isolates and it was scored high compared to other pathogens. This fungus can infect any part of the plant and the variability among isolates is well documented. The aggressiveness depends on the environment interaction among other factors.

\section{V.Conclusion and recommendations}

Four root rot diseases were identified and characterized including Rhizoctoniaspp,
Fusariumspp, Sclerotiumspp and several Pythium spp. Morphological characterization showed that Fusarium spp, Rhizoctonia spp and Sclerotium spp were predominant than Pythium species. The four diseased were observed especially in northern and southern parts of Rwanda than east and western parts of Rwanda. The collected and characterized isolates constitute an important resource for screening and improvement of dry bean germoplasm in Rwanda. Fusarium was identified as the most predominant fungal pathogen in Rwanda. It was observed that Sclerotium was the most virulent pathogens since most of its isolates scored high compared to other pathogens. The selected isolates are highly recommended to be used in selecting breeding materials in Rwanda. The total bean root rot disease control program is recommended to reduce losses associated with these diseases especially the use of resistant varieties since the fungi are widespread and survive in soils for a long time. It is highly recommended to promote the use of integrated technologies to reduce bean root rot causing pathogens especially Sclerotium. Further studies are recommended to characterize the identified pathogens.

\section{Acknowledgement}

The author would like to acknowledge the contribution of Fred Kato from CIAT Uganda, Annuarite Uwera, Vedaste Ahishakiye, Nyirahategekima Esperance, the bean team of RAB, laboratory staff of RAB and all other scientists who contributed to this work. We are grateful to Rwanda Agriculture Board, CIAT Africa, NIFA project for all the support to this work.

\section{References}

[1] Asare-Marfo, D., Birol, Katsvairo,E.L., Manirere, J.A., Maniriho, F. and Roy D. (2004) "Farmer Choice of Bean Varieties in Rwanda: Lessons learnt for HarvestPlus Delivery and Marketing Strategies." HarvestPlus.CIAT (2004). Enhancing farmers' access to seed of improved bean varieties in Rwanda, CIAT, pp. 2. 
[2] Bayuelo-Jimenez,J.S. Debouck, D.G. and Lynch J. P. (2003) Growth, gas exchange, water relations, and ion composition of Phaseolus species grown under saline conditions. Field Crop Research 80:207222.doi:10.1016/S0378-4290(02)00179-X

[3] Beebe, S.; Gonzalez, A.V.; Rengifo, J. (2000). Research on trace minerals in common bean. Food. Nutr.Bull (21): 387391.

[4] Binagwa, P.H. , Bonsi,C.K., Msolla, S. N. and Ritte, I. I. (2015). Morphological and molecular identification of Pythium spp. isolated from common beans (Phaseolus vulgaris) infected with root rot disease. African Journal of Plant Science. Vol. 10(1), pp. 1-9, DOI: 10.5897/AJPS2015.1359 ( http://www.academicjournals.org/AJPS)

[5] Blair, M., Gonzales,L.F., Kimani, P.M.; Butare, L. (2010) Genetic diversity, intergene pool introgression and nutritional quality of common beans (Phaseolus vulgaris L.) from central africa. Theor. Appl. Genet. 2010, 121, 237-248.

[6] Buruchara, R., Estévez de Jensen, C., Godoy, G., Abawi, G., Pasche, J.and Lobo Junior, M., Mukankusi, C. (2015). A review of the root rot diseases of common bean with emphasis in LatinAmerica and AfricaCommon Bean Disease on AngularLeaf Spot and Root Rot oral paper presentation in the conference.Protea Hotel Kruger Gate Skukuza, South Africa July 2023 , 2015

(http://arsftfbean.uprm.edu/bean/wpcontent/uploads/2015/09/BDW-Day2-RobinBuruchara.pdf .

[7] Chikoti, M. (2016). "Morphological and Molecular Identification and Characterization of Dry Bean Fungal Root Rot Pathogens in Zambia" (2016).Theses, Dissertations, and Student Research in Agronomy and Horticulture.Paper 112. http://digitalcommons.unl.edu/agronhortdiss/ 112

[8] Chilvers, M. and Hanson, L.(2015). Soil Borne Diseases of Dry Bean, avalaible on line http://www.fieldcroppathology.msu.edu, accessed in May 2015CIAT-AFrica. Highlights. CIAT, 1-2.

[9] CIAT, (2008). Farm-level impacts of improved bean varieties and agronomic technologies in Rwanda'Highlights, CIAT in Africa, no.41, Kampala: CIAT

[10] Graham, P.H., Rosas J.C., de Jensen, E.C., Peralta, E., B., lustyT., Acosta-Gallegos, J., Arraes Pereira, P. (2003). Addressing edaphic constraints to bean production:the bean/cowpea CRSP project in perspective. Field Crop Research 82, 179-192.

[11] Larochelle, C.,Alwang, J., Norton, G.W., Katungi,E. and Labarta,R.A. (2014) "Ex-Post Impact of Adopting Improved Bean Varieties on Poverty and Food Security in Uganda and Rwanda."Selected Paper prepared for presentation at the Agricultural \& Applied Economics Association's2014 AAEA Annual Meeting, Minneapolis, MN, July 2729, 2014.

[12] Larochelle, C. and Alwang J. (2014). Impacts of Improved Bean Varieties on Food Security in Rwanda.Selected Paper prepared for presentation at the Agricultural \& Applied Economics Association's2014 AAEA Annual Meeting, Minneapolis, MN, July 2729,

2014. http://www.Impacts $\% 20$ of $\% 20$ Improved $\% 20$ Bean\%20Varieties\%20on\%20Food\%20Secu rity\%20in\%20Rwanda_AAEA.pdf

[13] Mukamuhirwa F. Mukankusi M.C., Tusiime, G., Butar L., Musoni A., Ngaboyisonga C., Gahakwa D., Gibson P. and Kelly K. (2017). Resistance Levels to Root Rot and Angular Leaf Spot Diseases in Selected High Iron Bean Genotypes. Journal of Advances in Crop Science and Technology. Adv. Crop Sci. Tech 5: 274. doi: 10.4172/2329-8863.1000274

[14] Nzungize, J.,Gepts P.,Buruchara,R., S. Buah, P. Ragama, J. P. Busogoro andJ. P. Baudoin. (2011). Pathogenic and molecular characterization of Pythiumspecies inducing root rot symptoms of common beanin Rwanda 
[15] Petry, N., Boy, E., Wirth J.P. and Hurrell,R.F. (2015) Review: The Potential of the Common Bean (Phaseolus vulgaris) as a Vehicle for Iron Biofortification . Nutrients 2015, 7, 1144-1173; doi:10.3390/nu7021144 (ISSN 2072-6643 www.mdpi.com/journal/nutrients)

[16] Rainey, K.M. and Griffiths, P.D.(2005). Inheritance of heat tolerance during reproductive development in snap bean (Phaseolus vulgaris L.). J. Am. Soc Hort. Sci., 130(5): 700-706
[17] Sharma, K.K. and Lavanya M. (2002).Recent developments in trans-genics for abiotic stress in legumes of the semi-arid tropics. JIR-CAS Working Report: 61-73

[18] Wortmann,C.S, Kirkby,R.A., Eledu,C.A. and Allen,D.J., (1998).Atlas of common bean (Phaseoulus vulgaris) production in Africa. International Center for Trocal Agriculture, cali, Colombia. 